\title{
The space of measurement outcomes as a spectrum for non-commutative algebras
}

\author{
Bas Spitters
}

\begin{abstract}
Bohrification defines a locale of hidden variables internal in a topos. We find that externally this is the space of partial measurement outcomes. By considering the $\neg \neg$-sheafification, we obtain the space of measurement outcomes which coincides with the spectrum for commutative $\mathrm{C}^{*}$-algebras.
\end{abstract}

\section{Introduction}

By combining Bohr's philosophy of quantum mechanics, Connes' non-commutative geometry [Con94], constructive Gelfand duality [BM00b, BM00a, Coq05, CS09] and inspiration from Doering and Isham's spectral presheaf [DI08], we proposed Bohrification as a spatial quantum logic [HLS09a, HLS09b]. Given a $C^{*}$-algebra $A$, modeling a quantum system, consider the poset of Bohr's classical concepts

$$
\mathscr{C}(A):=\left\{C \mid C \text { is a commutative } \mathrm{C}^{*} \text {-subalgebra of } A\right\} .
$$

In the functor topos Sets ${ }^{\mathscr{C}(A)}$ we consider the Bohrification $\underline{A}$ : the trivial functor $C \mapsto C$. This is an internal $C^{*}$-algebra of which we can compute the spectrum, an internal locale $\Sigma$ in the topos Sets ${ }^{\mathscr{C}(A)}$. This locale, or its externalization, is our proposal for an intuitionistic quantum logic [HLS09a, HLS09b].

In the present paper we explore a possible refinement of this proposal motivated by what happens for commutative algebras and by questions about maximal subalgebras.

In section 4 we compute the externalization of this locale. It is the space of partial measurement outcomes: the points are pairs of a $\mathrm{C}^{*}$-subalgebra together with a point of its spectrum. This construction raises two natural questions:

- Can we restrict to the maximal commutative subalgebras, i.e. total measurement frames?

- Are we allowed to use classical logic internally?

In section 5 we will see that, in a sense, the answers to both of these questions are positive. The collection of maximal commutative subalgebras covers the space in the dense topology and this dense, or double negation, topology forces (sic) the logic to be classical. By considering the $\neg \neg$-sheafification, we obtain a genuine generalization of the spectrum. Moreover, our previous constructions [HLS09a] of the phase space $(\Sigma)$ and the state space still apply essentially unchanged.

\section{Preliminaries}

An extensive introduction to the context of the present paper can be found in [HLS09b, HLS09a] and the references therein. Here we will just repeat the bare minimum of definitions.

A site on an poset defines a covering relation. To simplify the presentation we restrict to the case of a meet-semilattice.

Definition 1 Let $L$ be a meet-semilattice. A covering relation on $L$ is a relation $\triangleleft \subset L \times P(L)$ satisfying:

S. B. Cooper, E. Kashefi, P. Panangaden (Eds.): Developments in

Computational Models (DCM 2010)

EPTCS 26, 2010, pp. 127-133 doi 10.4204/EPTCS.26.12 
1. if $x \in U$ then $x \triangleleft U$;

2. if $x \triangleleft U$ and $U \triangleleft V$ (i.e. $y \triangleleft V$ for all $y \in U$ ) then $x \triangleleft V$;

3. if $x \triangleleft U$ then $x \wedge y \triangleleft U$;

4. if $x \triangleleft U$ and $x \triangleleft V$, then $x \triangleleft U \wedge V$, where $U \wedge V=\{x \wedge y \mid x \in U, y \in V\}$.

Such a pair $(L, \triangleleft)$ is called a formal topology.

Every formal topology defines a locale, conversely every locale can be presented in such a way.

Definition 2 Let $(L, \triangleleft)$ be a formal topology. A point is an inhabited $\alpha \subset L$ that is filtering with respect to $\leq$, and such that for each $a \in \alpha$ if $a \triangleleft U$, then $U \cap \alpha$ is inhabited. In short, it is a completely prime filter.

The spectrum $\Sigma$ of a $\mathrm{C}^{*}$-algebra $A$ can be described directly as a lattice $L(A)$ together with covering a relation; see [CS09].

\section{Measurements}

In algebraic quantum theory [Emc84, Haa96, Lan98], a measurement is a (maximal) Boolean subalgebra of the set of projections of a von Neumann algebra. The outcome of a measurement is the consistent assignment of either 0 or 1 to each element (test, proposition) of the Boolean algebra: the outcome is an element of the Stone spectrum. Unlike von Neumann-algebras, $\mathrm{C}^{*}$-algebras need not have enough projections. It is customary to replace the Boolean algebra by a commutative $\mathrm{C}^{*}$-subalgebra and the Stone spectrum by the Gelfand spectrum. Although a detailed critique of the measurement problem is beyond the scope of this paper, with the previous motivation we will make the following definition.

Definition 3 A measurement outcome is a point in the spectrum of a maximal commutative subalgebra.

The choice to restrict to maximal subalgebras varies between authors. The present choice fits with our presentation.

\section{The space of partial measurement outcomes}

Iterated topos constructions, similar to iterated forcing in set theory were studied by Moerdijk [Moe86][Joh02, C.2.5]. To wit, let $\mathscr{S}$ be the ambient topos. One may think of the topos Sets, but we envision applications where a different choice for $\mathscr{S}$ is appropriate [HLS09b].

Theorem 4 (Moerdijk) Let $\mathbb{C}$ be a site in $\mathscr{S}$ and $\mathbb{D}$ be a site in $\mathscr{S}[\mathbb{C}]$, the topos of sheaves over $\mathbb{C}$. Then there is a site $1 \mathbb{C} \ltimes \mathbb{D}$ such that

$$
\mathscr{S}[\mathbb{C}][\mathbb{D}]=\mathscr{S}[\mathbb{C} \ltimes \mathbb{D}] .
$$

We will specialize to sites on a poset and without further ado focus on our main example. As before, let

$$
\mathscr{C}(A):=\left\{C \mid C \text { is a commutative } C^{*} \text {-subalgebra of } A\right\} .
$$

\footnotetext{
${ }^{1}$ The notation $\ltimes$ is motivated by the special case where $\mathbb{C}$ is a group $G$ considered as a category with one object and $\mathbb{D}$ is a group $H$ in Sets $^{G}$. Then $\mathbb{C} \ltimes \mathbb{D}$ is indeed the semi-direct product $H \ltimes G$
} 
Let $\mathbb{C}:=\mathscr{C}(A)^{\text {op }}$ and $\mathbb{D}=\Sigma$ the spectrum of the Bohrification, we compute $\mathbb{C} \ltimes \mathbb{D}$. The objects are pairs $(C, u)$, where $C \in \mathscr{C}(A)$ and $u$ in $L(C)$. Define the order $(D, v) \leq(C, u)$ as $D \supset C$ and $v \subset u$. In terms of forcing, this is the information order and the objects are forcing conditions. We add a covering relation $(C, u) \triangleleft\left(D_{i}, v_{i}\right)$ as for all $i, C \subset D_{i}$ and $C \Vdash u \triangleleft V$, where $V$ is the pre-sheaf generated by the conditions $D_{i} \Vdash v_{i} \in V$. It follows from the general theory that this is a Grothendieck topology.

We simplify: the pre-sheaf $V$ is generated by the conditions $D_{i} \Vdash v_{i} \in V$ means

$$
V(D):=\left\{v_{i} \in V(D) \mid D \supset D_{i}\right\}
$$

Hence,

$$
C \Vdash u \triangleleft V \text { iff } u \triangleleft\left\{v_{i} \mid D_{i}=C\right\}
$$

by the following lemma.

Lemma 5 [HLS09a] Let $V$ be an internal sublattice of $L$. Then $C \Vdash u \triangleleft V$ iff $u \triangleleft V(C)$.

Definition 6 A partial measurement outcome is a point in the spectrum of a commutative subalgebra. A consistent ideal of partial measurement outcomes is a family $\left(C_{i}, \sigma_{i}\right)$ of partial measurement outcomes such that the $C_{i}$ are an ideal in $\mathscr{C}(A)$ and if $C_{i} \subset C_{j}$, then $\sigma_{i}=\left.\sigma\right|_{C_{j}}$.

Theorem 7 The points of the locale generated by $\mathbb{C} \ltimes \mathbb{D}$ are consistent ideals of partial measurement outcomes.

Proof Let $\tau$ be a point, that is a completely prime filter. Suppose that $(D, u) \in \tau$, then by the covering relation for $\mathbb{D}, \tau$ defines a point of the spectrum $\Sigma(D)$. This point is defined consistently: If $u \in L(C) \subset$ $L(D)$, then $(D, u) \leq(C, u)$. Hence, if $(D, u) \in \tau$, so is $(C, u)$ and the point in $\Sigma(D)$ defines a point in $\Sigma(C)$ as a restriction of functionals. When both $(C, u)$ and $\left(C^{\prime}, u^{\prime}\right)$ are in $\tau$, then, by directedness, there exists $(D, v)$ in $\tau$ such that $C, C^{\prime} \subset D$ and $v \subset u, u^{\prime}$. Moreover, $(C, u) \in \tau$ implies $(C, \top) \in \tau$, now the set $\{C \mid(C, \top) \in \tau\}$ is directed and downclosed.

Conversely, let $\left(C_{i}, \sigma_{i}\right)$ be a consistent ideal of partial measurement outcomes, then

$$
\mathscr{F}:=\left\{\left(C_{i}, u\right) \mid \sigma_{i} \in u\right\}
$$

defines a filter: it is up-closed and lower-directed. Suppose that $\left(C_{i}, u\right) \triangleleft\left(A_{j}, v_{j}\right)$, that is $j, C \subset A_{j}$ and $A_{k} \Vdash u \triangleleft V$, where $V=\left\{v_{j} \mid A_{j}=A_{k}\right\}$. Then $\left(A_{k}, v_{j}\right) \in \mathscr{F}$, because $\sigma_{k}$ is a point/completely prime filter.

It is tempting to identify the ideal of partial measurement outcomes with its limit. However, the ideal and its limit define different points. These points are identified in Section 5 .

Let us call this locale $p M O$ for (consistent ideals of) partial measurement outcomes. For commutative $\mathrm{C}^{*}$-algebras $p M O$ is similar, but not equal, to the spectrum:

Corollary 8 For a compact regular $X$, the points of $p M O(C(X))$ are points of the spectrum of a $C^{*}$ subalgebra of $C(X)$.

An explicit external description of the locale may be found in [HLS09b]. The present computation gives an alternative description which makes it easy to compute the points. 


\section{Maximal commutative subalgebras, classical logic and the spectrum}

As stated in the introduction, we address the following questions:

- Can we restrict to the maximal commutative subalgebras?

- Are we allowed to use classical logic internally?

In a sense, the answers to both of these questions are positive. The collection of maximal commutative subalgebras covers the space $\mathscr{C}(A)$ in the dense topology and this dense, or double negation, topology forces the logic to be classical.

Sheaves for the dense topology may be used to present classical set theoretic forcing or Boolean valued models. In set theoretic forcing one considers the topos $\operatorname{Sh}(P, \neg \neg)$ [MM92, p.277]. The dense topology on a poset $P$ is defined as $p \triangleleft D$ if $D$ is dense below $p$ : for all $q \leq p$, there exists a $d \in D$ such that $d \leq q \mathrm{I}^{2}$ The locale presented by this site is a Boolean algebra, the topos is a Boolean valued model. If $P$ is directed and $I$ is an ideal in $P$, the directed join is contained in the double negation of $I$. Hence the double negation topology is coarser than the $j$-topology described above.

This topos of $\neg \neg$-sheaves satisfies the axiom of choice [MM92, VI.2.9] when our base topos does. The associated sheaf functor sends the presheaf topos $\hat{P}$ to the sheaves $\operatorname{Sh}(P, \neg \neg)$. The sheafification can be described explicitly [MM92, p.273] for $V \longmapsto W$ :

$$
\neg \neg V(p)=\{x \in W(p) \mid \text { for all } q \leq p \text { there exists } r \leq q \text { such that } x \in V(r)\} .
$$

We apply this to the poset $\mathscr{C}(A)$. We write $A$ for the constant functor $C \mapsto A$. Then $\underline{A} \subset A$ in $\operatorname{Sets}^{\mathscr{C}(A)}$.

For commutative $A, \mathscr{C}(A)$ has $A$ as bottom element. For all $C, \underline{A}_{\neg\urcorner}(C)=A$.

For the general case, we observe that each $C$ is covered by the collection of all its supersets. By Zorn? 3 , each commutative subalgebra is contained in a maximal commutative one. Hence the collection of maximal commutative subalgebras is dense. So, $\underline{A}_{\neg \neg}(C)$ is the intersection of all maximal commutative subalgebras containing $C$.

The covering relation for $(\mathscr{C}(A), \neg \neg) \ltimes \underline{\Sigma}$ is $(C, u) \triangleleft\left(D_{i}, v_{i}\right)$ iff $C \subset D_{i}$ and $C \Vdash u \triangleleft V_{\neg \neg}$, where $V_{\neg\urcorner}$ is the sheafification of the presheaf $V$ generated by the conditions $D_{i} \Vdash v_{i} \in V$. Now, $V \longmapsto L$, where $L$ is the spectral lattice of the presheaf $\underline{A}$.

$$
V_{\neg\urcorner}(C)=\{u \in L(C) \mid \forall D \leq C \exists E \leq D . u \in V(E)\} .
$$

So, $(C, u) \triangleleft\left(D_{i}, v_{i}\right)$ iff

$$
\forall D \leq C \exists D_{i} \leq D . u \triangleleft V\left(D_{i}\right) .
$$

Theorem 9 The locale $M O$ generated by $(\mathscr{C}(A), \neg \neg) \ltimes \underline{\Sigma}$ classifies measurement outcomes. It is a (dense) sublocale of pMO.

Proof In the context of Theorem 7 we suppose that $(C, \top) \in \tau$. The subalgebra $C$ is covered by all the maximal commutative subalgebras containing it, so by directedness we conclude that $(M, \top) \in \tau$ for some maximal $M$.

The $M O$ construction is a non-commutative generalization of the spectrum. In this sense it behaves better then $P M O$; compare Corollary 8 .

\footnotetext{
${ }^{2}$ Constructively, this also defines a topology [Coq09]. However, we need classical logic to prove that it coincides with the double negation topology.

${ }^{3}$ Here we use classical meta-logic.
} 
Corollary 10 For a compact regular $X, X \cong M O(C(X))$.

Proof $C(X)$ is the only maximal commutative subalgebra of $C(X)$.

By considering the double negation we may use classical logic internally in our Boolean valued model.

The Kochen-Specker theorem can be reformulated as the non-existence of certain global sections [DI08, HLS09b]. This connection carries over essentially unchanged, since a global section of a sheaf is (by definition) a global section of the sheaf considered as a presheaf.

Theorem 11 Kochen-Specker: Let $H$ be a Hilbert space with $\operatorname{dim} H>2$ and let $A=B(H)$. Then the $\neg \neg$-sheaf $\sum$ does not allow a global section.

Internally the axiom of choice holds, so $\Sigma$ is a compact Hausdorff space. Still spectrum does not have a global point and the algebra does not have a global element.

As an example, consider the matrix algebra $M_{n}$. Let $D_{n}$ be the $n$-dimensional diagonal matrix. The maximal subalgebras of $M_{n}$ are $\left\{\varphi D_{n} \mid \varphi \in S U_{n}\right\}$; see [CHLS09]. Moreover, $\Sigma \cong\{1, \ldots, n\}$ in $\mathrm{Sh}(\mathscr{C}(A), \neg \neg)$. This is a complete Boolean algebra. We have arrived at the setting of iterated forcing as in set theory. Iterated forcing in set theory may be presented as follows; see Moerdijk [Moe86, Ex 1.3a]. If $P$ is a poset in Sets, and $Q$ is a poset in $\hat{P}$, then $P \ltimes Q$ is the poset in Sets of pairs $(p, q)$ with $p \in P$, $p \Vdash q \in Q$, and $(p, q) \leq\left(p^{\prime}, q^{\prime}\right)$ iff $p \leq p^{\prime}$ and $p \Vdash q \leq q^{\prime}$. If $\mathscr{E}=\operatorname{Sh}(P, \neg \neg)$, and $Q$ is a poset in $\mathscr{E}$, $\mathscr{F}:=\operatorname{Sh}_{\mathscr{E}}(Q, \neg \neg)$, then $\mathscr{F} \cong \operatorname{Sh}(P \ltimes Q, \neg \neg)$. In other words, $(P, \neg \neg) \ltimes(Q, \neg \neg) \cong(P \ltimes Q, \neg \neg)$. If, as in the case of $M_{n}, Q$ is a cBA in $\mathscr{E}$, then $(Q, \neg \neg) \cong Q$. So $(P \ltimes Q, \neg \neg) \cong(P, \neg \neg) \ltimes Q$. We expect similar simplifications when starting from a Rickart $C^{*}$-algebra [HLS09c].

A similar $\neg \neg$-transformation can be applied to our Bohrification of OMLs. In the example studied in [HLS09b], we compute a 17 element Heyting algebra from an OML. Adding the double negation we obtain a 16 element Boolean algebra. The function $f(0)=0$ and $f(i)=1$ is 'eventually' equal to the constant function 1. As a result, we obtain the product of 4 Boolean algebras, the spectrum is the coproduct of the corresponding locales.

\section{Conclusions and further research}

We have presented a non-commutative generalization of the spectrum motivated by physical considerations.

We suggest another way to restrict to maximal subalgebras, while preserving the possibility to compute a unique functional from a global section. Consider a matrix algebra. Let $p \in C$ be a projection and suppose that $M \mapsto \sigma_{M} \in \Sigma(M)$ is continuous with respect to the unitary group action. Then $\sigma(p) \in\{0,1\}$, say it is 0 . Since the unitary group is connected and acts transitively on the maximal subalgebras, $\sigma\left(u^{*} p\right)=0$ for all $u$. Suppose that $p \in M_{1}, M_{2}$. Let $u$ transform $M_{1}$ into $M_{2}$, but leave $p$ fixed. We see that $\sigma(p)=0$ independent of the choice of maximal subalgebra. By linearity and density, this extends from projections to general elements: $\sigma$ may be uniquely defined on all elements. This suggests that, at least for matrix algebras, the independence guaranteed by the poset, may also be guaranteed by the group action. We leave this issue to future research.

Bohrification, i.e. the $p M O$ construction, is not functorial when we equip $\mathrm{C}^{*}$-algebras with their usual morphisms. The construction is functorial when we change the notion of morphism [vdBH10]. More work seems to be need for the $M O$ construction: We have $\left(I_{2}, \top\right) \triangleleft(C(2),\{(0,1),(1,0)\})$. However, this no longer holds when we map $C(2)$ into $M_{2}$. In short, covers need not be preserved under natural notions of morphism. 
Bohrification may be described as a (co)limit [vdBH10]. While technically different the intuitive meaning is similar: we are only interested in what happens eventually.

As in [HLS09a, HLS09b] treat $\mathscr{C}(A)$ as a mere poset. However, at least in the finite dimensional case, this poset has an interesting manifold structure [HLS09b]. Escardo [Esc98] provides a construction of the support of a locale which often coincides with its maximal points. It may be possible to use this construction to refine the present results by maintaining the topological structure.

\section{Acknowledgements}

I would like to thank Chris Heunen, Klaas Landsman and Steve Vickers for discussions and comments on a draft of this paper. I thank the referees for their comments.

\section{References}

[BM00a] Bernhard Banaschewski and Christopher J. Mulvey. The spectral theory of commutative $\mathrm{C}^{*}$-algebras: the constructive Gelfand-Mazur theorem. Quaestiones Mathematicae, 23(4):465-488, 2000.

[BM00b] Bernhard Banaschewski and Christopher J. Mulvey. The spectral theory of commutative $\mathrm{C}^{*}$-algebras: the constructive spectrum. Quaestiones Mathematicae, 23(4):425-464, 2000.

[CHLS09] Martijn Caspers, Chris Heunen, Klaas Landsman, and Bas Spitters. Intuitionistic quantum logic of an n-level system. Foundations of Physics, 39(7):731-759, 2009.

[Con94] Alain Connes. Noncommutative geometry. Academic Press Inc., San Diego, CA, 1994.

[Coq05] Thierry Coquand. About Stone's notion of spectrum. Journal of Pure and Applied Algebra, 197:141158, 2005.

[Coq09] Thierry Coquand. About goodman's theorem. manuscript, 2009.

[CS09] Thierry Coquand and Bas Spitters. Constructive Gelfand duality for $\mathrm{C}^{*}$-algebras. Mathematical Proceedings of the Cambridge Philosophical Society, 2009. Accepted.

[DI08] Andreas Döring and Chris J. Isham. 'What is a thing?': Topos theory in the foundations of physics. arXiv:0803.0417, 2008.

[Emc84] Gérard G. Emch. Mathematical and conceptual foundations of 20th-century physics, volume 100 of North-Holland Mathematics Studies. North-Holland Publishing Co., Amsterdam, 1984. Notas de Matemática [Mathematical Notes], 100.

[Esc98] M.H. Escardó. Properly injective spaces and function spaces. Topology and its Applications, 89(12):75-120, 1998.

[Haa96] Rudolf Haag. Local quantum physics. Texts and Monographs in Physics. Springer-Verlag, Berlin, second edition, 1996. Fields, particles, algebras.

[HLS09a] Chris Heunen, Klaas Landsman, and Bas Spitters. A topos for algebraic quantum theory. Communications in mathematical physics, 291(1):63-110, 2009.

[HLS09b] Chris Heunen, Nicolaas P. Landsman, and Bas Spitters. Bohrification. In Hans Halvorson, editor, Deep Beauty. 2009. to appear.

[HLS09c] Chris Heunen, Nicolaas P. Landsman, and Bas Spitters. Bohrification of operator algebras and quantum logic. Synthese, 2009. Accepted for publication, preprint available at http://arxiv.org/abs/ 0905.2275

[Joh02] Peter T. Johnstone. Sketches of an Elephant: A topos theory compendium, volume 1. Clarendon Press, 2002. 
[Lan98] Nicolaas P. Landsman. Mathematical topics between classical and quantum mechanics. Springer, 1998.

[MM92] Saunders Mac Lane and Ieke Moerdijk. Sheaves in Geometry and Logic. Springer, 1992.

[Moe86] Ieke Moerdijk. Continuous fibrations and inverse limits of toposes. Compositio Math., 58(1):45-72, 1986.

[vdBH10] Benno van den Berg and Chris Heunen. Noncommutativity as a colimit. 2010. Preprint available at arXiv:1003.3618v1. 